\title{
Linking Changes in Heart Rate Variability to Mood Changes in Daily Life
}

\author{
Oliver Carr ${ }^{1}$, Fernando Andreotti ${ }^{1}$, Kate E A Saunders ${ }^{2,3}$, Amy C Bilderbeck ${ }^{2}$, Guy M Goodwin ${ }^{2,4}$, \\ Maarten De Vos ${ }^{1,4}$ \\ ${ }^{1}$ Institute of Biomedical Engineering, Oxford, UK \\ ${ }^{2}$ Department of Psychiatry, Oxford, UK \\ ${ }^{3}$ Oxford Health NHS Foundation Trust, Oxford, UK \\ ${ }^{4}$ Sleep and Circadian Neuroscience Institute, Oxford, UK
}

\begin{abstract}
Background: Dynamic changes of heart rate are considered as markers for autonomic nervous system (ANS) balance and potentially mood. Objective markers of mood are especially of interest when monitoring mental health conditions such as bipolar disorder $(B D)$ and borderline personality disorder (BPD).

Methods: Ambulatory ECG recordings, actigraphy and mood recordings were collected from $13 \mathrm{BD}, 10 \mathrm{BPD}$ and 15 healthy participants. A total of 2465 -minute segments of ECG with a corresponding mood entry and actigraphy recording were collected. Heart rate variability (HRV) measures were calculated for each segment.

Results: Significant effects were found for changes in positive mood on changes in HRV for BD participants $\left(R^{2}=0.076, p=0.009\right)$ for mean $R-R$ interval $(m R R)$ and $\left(R^{2}=0.061, p=0.026\right)$ for high frequency $(H F)$ frequency power. Similar effects were found for BPD participants for negative mood on $m R R\left(R^{2}=0.126, p=0.014\right)$ and on negative mood and $H F\left(R^{2}=0.81, p=0.049\right)$.

Conclusions: Objective measures, such as $H R V$, which are shown to be linked to changes in mood could allow better understanding of the relationship between mood and cardiovascular behaviour in mental health disorders. In the future, such metrics could help management of these conditions through adjustments in treatments.
\end{abstract}

\section{Introduction}

Mental health disorders such as bipolar disorder (BD) and borderline personality disorder (BPD) are associated with variable mood [1] and with dysregulation of the autonomic nervous system (ANS) through differing levels of vagal tone $[2,3]$. Fluctuations in heart rate (HR) are due to complex non-linear interactions between the brain and heart, thus heart rate variability (HRV) measures can be used to quantify ANS activity [4]. A reduction in HRV can indicate problems with self-regulation, stress or other pathologies, whereas as increased variability is considered healthy [5].

A wide range of studies have been carried out which investigate HRV metrics in BD patients, with the majority performed under controlled environments in a short space of time [6]. Meta-analyses and reviews of the literature conclude HRV is reduced in BD and there is dysregulation of the ANS [7]. There are far fewer studies which measure HRV in BPD, however results indicate HRV is reduced in a similar manner [8]. These studies all show global differences in HRV but do not show how HRV changes in mental health disorders under different conditions or emotional states.

Associations between HRV measures and moods have been investigated in various controlled environments. A study on healthy participants measured resting HRV and retrospective questionnaires found a negative relationship between high frequency (HF) power and perceived stress [9]. Another study measured resting HRV metrics and questionnaires on affect and found a similar relationship between HF power and negative affect [10]. A summary of the literature on ANS activity in emotion reports increased HR, HF power and reduced low frequency (LF) to HF ratio (LF:HF) with increased anxiety, as well as increased HR with increased sadness across varying participant groups [11]. It is also reported that increased $\mathrm{HR}$, reduced HF power and no change in LF power is associated with increased happiness. However, there is large variation in the methods and the reported results in these studies, with varying mood and stimulated emotion showing varying associations between HRV and emotion. Moreover, although these studies indicate HRV is altered in these patient groups, they do not show how HRV behaves during natural mood changes during daily life.

In order to evaluate more natural links between HRV and mood, ambulatory recordings of HRV during daily life along with regular moods reports are required. A study [12] investigated two to three days of ECG recordings from healthy subjects and mood questionnaires collected every hour, with correlations 
found between HRV and mood in daily life. Positive correlations were found between changes in $\mathrm{HR}$ and changes in vigour, whereas negative correlations were found between changes in the root mean of successive differences of R-R intervals and vigour and changes in $\mathrm{HF}$ power and vigour.

Here, we show how HRV measures change with mood over short time periods when recorded in daily life and how these changes differ between BD participants, BPD participants and healthy controls. Such continuous information would be valuable to monitor BD and BPD patients and adjust treatment to avoid patients entering negative mood states.

\section{Methods}

\subsection{Data collection}

The data was collected as part of the Automated Monitoring of Symptom Severity (AMoSS) study at the University of Oxford. The study was approved by the NRES Committee East of England - Norfolk (13/EE/0288). Further description of the study can be found in previous works [13-15].

Participants wore a mobile ECG (Shimmer Sensing) device, recording at $256 \mathrm{~Hz}$ and an actigraphy accelerometer (Proteus) on their torso between 12 and 72 hours. They were prompted to self-evaluate six moods (Anxious, Elated, Sad, Angry, Energetic and Irritable) through a mobile phone app on a seven point Likert scale ten times evenly spaced throughout each day.

Data was collected from 13 BD participants, 10 BPD and 15 healthy controls. The demographics of the groups were similar, with a mean age of $38.18 \pm 11$.80years and a mean BMI of $26.51 \pm 4.92 \mathrm{~kg} / \mathrm{m}^{2}$. However there were differences between genders, with $5 / 8$ male/female BD participants, 1/9 for BPD participants and 4/11 for healthy controls. In order to determine how HRV changes with mood, it is important to remove activity levels as a confounding factor. Based on [12], in this study all ECG segments with the corresponding acceleration data having a mean value over $30 \mathrm{mG}$ on the preceding 10 minutes were excluded from the analysis.

A total of 246 five minute valid segments of ECG with corresponding actigraphy and a mood entry were collected from these participants.

\subsection{Heart rate variability calculation}

For each recording, QRS peaks were detected using traditional widely available algorithms. Visual inspection and manual correction were performed to avoid misclassifications. Well known HRV measures were calculated from the R-R interval series, with time domain and frequency domain measures shown in Table 1.
Table 1. HRV measures calculated and their physiological interpretations.

\begin{tabular}{ccc}
\hline Measure & & Interpretation \\
\hline Mean R-R interval & mRR & Heart rate \\
$\begin{array}{c}\text { Standard deviation of } \\
\text { R-R intervals }\end{array}$ & SDNN & Variability \\
Root mean squared & RMSSD & Variability \\
of successive & & \\
differences & & \\
High frequency & HF & $\begin{array}{c}\text { Parasympathetic } \\
\text { activity }\end{array}$ \\
$\begin{array}{c}\text { (0.15-0.4Hz) power } \\
\text { Low frequency }\end{array}$ & LF & $\begin{array}{c}\text { ANS activity } \\
\text { (0.04-0.15Hz) power }\end{array}$ \\
Low to high & LF:HF & $\begin{array}{c}\text { Sympathovagal } \\
\text { balance }\end{array}$ \\
\hline
\end{tabular}

\subsection{Mood analysis}

As self-reported absolute values for mood ratings are inherently subjective, differences between consecutive mood reports were used. As in [12], HRV measures were normalised by participant, the minimum value of each participant was subtracted from the rest of their measures before being divided by the range of their HRV measures. The time difference between successive recordings of HRV and mood varies greatly, especially across a night. To standardise the time differences, changes in mood and HRV were only calculated when the recordings were between one and three hours apart.

The first three principal components of the mood entries, negative, positive and irritable mood, were used in the analysis [14].

In order to account for inter-subject variability, a linear mixed effects model was used to calculate $\mathrm{R}^{2}$ values and corresponding p-values between each pair of mood changes (negative, positive and irritable) and the changes in HRV measures in Table 1.This accounts for variation in gradient and intercept with the effect of mood in individual participants.

\section{Results}

In Figure 1, the effects of HRV and positive mood changes are depicted. Figure 1 (a), (d) and (g) show the statistically significant relationships found in BD participants between changes in positive mood and $\mathrm{mRR}$, HF power and LF:HF respectively. Figure 1 (c) shows the statistically significant relationship between changes in positive mood and change in $\mathrm{mRR}$ in healthy controls.

In BPD participants, effects were found for changes in negative mood, depicted in Figure 2. The relationship between changes in negative mood and changes in mRR and HF power can be seen in Figure 2 (b) and (e). 


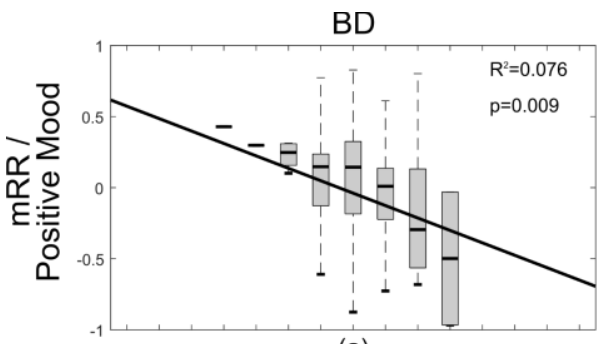

(a)

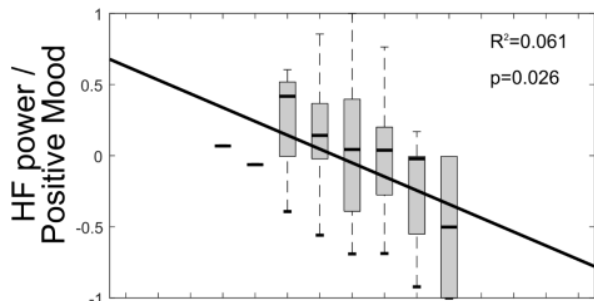

(d)

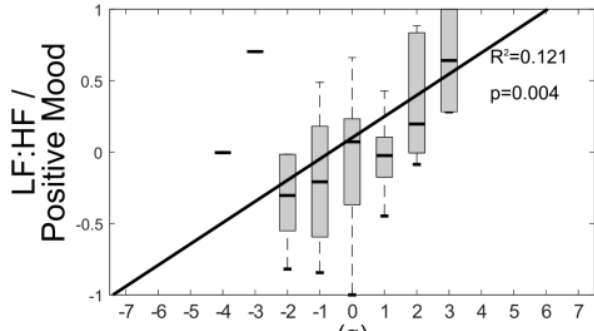

(g)

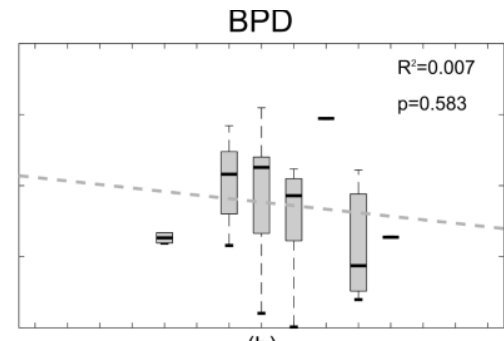

(b)

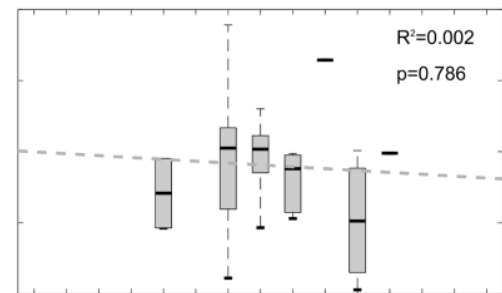

(e)

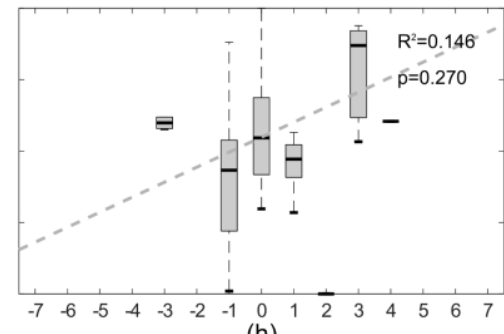

(h)

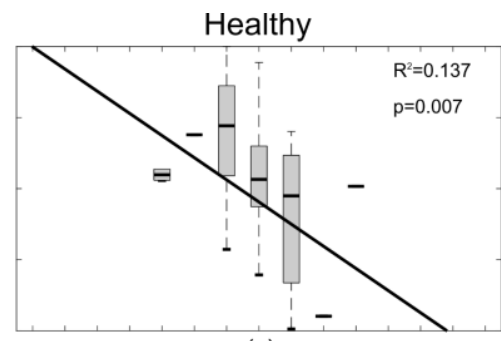

(c)

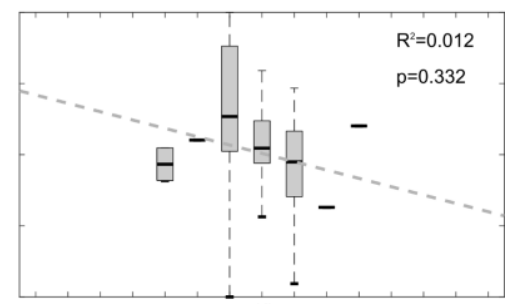

(f)

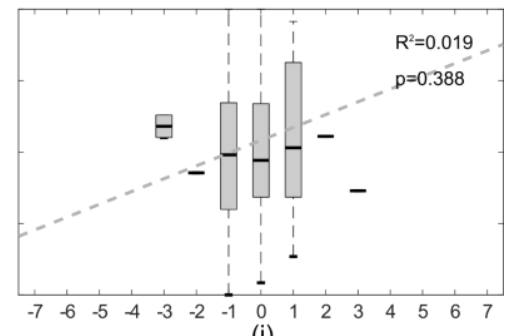

(i)

Change in Mood

Figure 1 - Statistically significant effects of mood on HRV are shown with a solid line. Significant effects were found in BD participants for: positive mood on $m R R\left(R^{2}=0.076, p=0.009\right)$ in (a), positive mood on HF power $\left(R^{2}=0.061, p=0.026\right)$ in $(d)$ and positive mood on LF:HF $\left(R^{2}=0.121, p=0.004\right)$ in $(\mathrm{g})$. A statistically significant effect was found for healthy control participants for changes in positive mood on changes in $\mathrm{mRR}\left(\mathrm{R}^{2}=0.137, \mathrm{p}=0.007\right)$ shown in $(\mathrm{c})$.

\section{Discussion}

HRV was linked to changes in mood in BD and BPD participants during daily life, with elimination of confounding factors such as activity and inter-patient variability. Similar results were described in the literature [9-11] with associations found between both HR and parasympathetic activity compared to positive and negative moods.

The significant effects found for BD participants were associated with positive mood. The negative relationship with mRR suggests HR increases as positive mood increases, with changes in mood explaining $7.6 \%$ of the variance. The negative trend with HF power suggests there is a reduction in parasympathetic activity with increasing positive mood. Changes in positive mood can also explain $12.1 \%$ of the variance in change in LF:HF power in BD. The only significant relationship found in healthy controls was also changes in positive mood on changes in mRR. These results agree with the literature [11] which reported increased HR, reduced HF power and unchanged LF power (thus increased LF:HF ratio) with an increase in happiness. Negative mood in BPD participants had a significant impact on $\mathrm{mRR}$, suggesting HR increases as moods become more negative. The HF power, thus parasympathetic activity, decreases with irritable mood and the LF:HF ratio increases as irritable mood increases.

The limitations of this study include medication of participants not being taken into account which could have effects on HRV. In addition, there are many external influences such as food, drink and smoking which also affect HRV measures, this may explain the low $\mathrm{R}^{2}$ values as variance in HRV is likely to be affected by these other factors. The effects of circadian rhythms have not been corrected for as there may be some natural variation in HRV measures throughout the day [15].

\section{Conclusion}

We have shown short term monitoring of ECG during daily life reveals changes in HRV can be associated with changes in mood, with HRV varying with positive mood changes in $\mathrm{BD}$, whereas HRV varies with negative mood changes in BPD. Finding objective markers of mood changes in mental health disorders could allow for better 


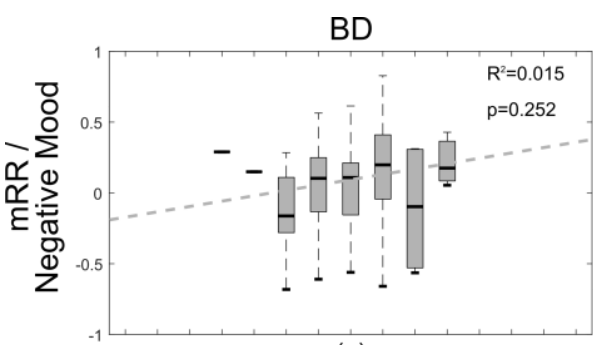

(a)

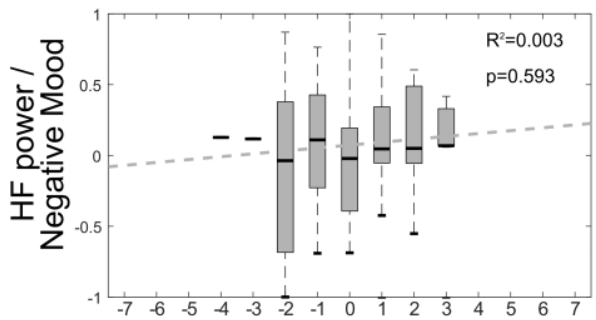

(d)

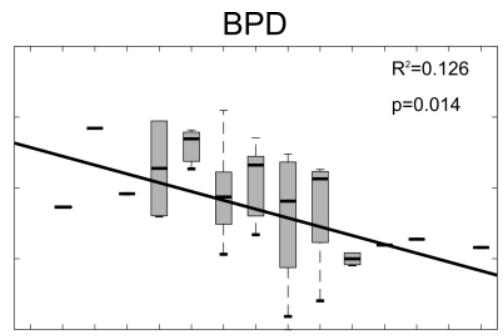

(b)

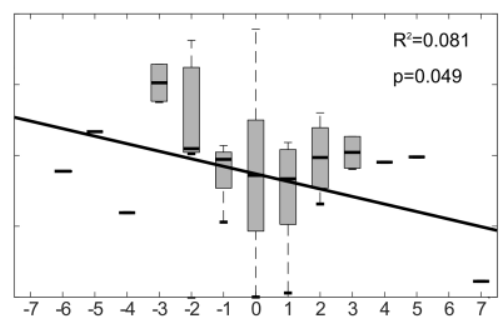

(e)

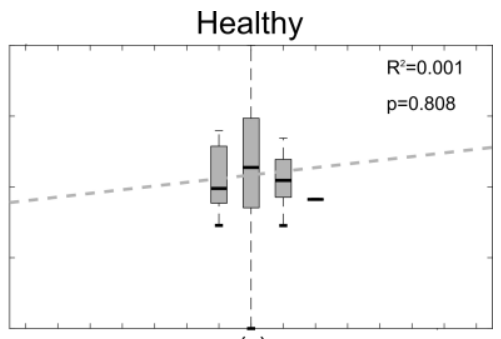

(c)

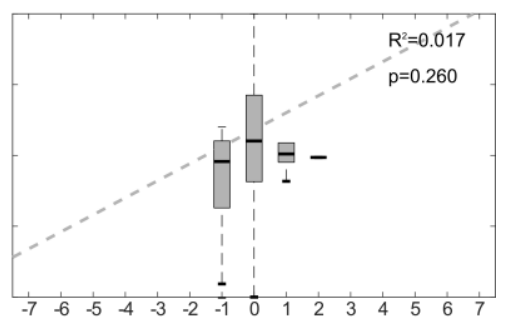

(f)

Change in Mood

Figure 2 - Statistically significant effects of moon on HRV are shown with a solid line. Significant effects were found in BPD participants for: negative mood on $\mathrm{mRR}\left(\mathrm{R}^{2}=0.126, \mathrm{p}=0.014\right)$ in (b) and negative mood on $\mathrm{HF}$ power $\left(\mathrm{R}^{2}=0.081, \mathrm{p}=0.049\right)$ in $(\mathrm{e})$.

monitoring of mood. This could allow for adjustments in treatments to be made in order to help prevent transitions into negative states.

\section{Acknowledgements}

This work was supported by EPSRC and Wellcome Trust Centre Grants 098461/Z/12/Z (Sleep, Circadian Rhythms and Neuroscience Institute) and 102616/Z (Collaborative Network for Bipolar Research to Improve Outcomes). OC is supported by the RCUK Digital Economy Programme EP/G036861/1 (Oxford Centre for Doctoral Training in Healthcare Innovation). FA is supported by the EPSRC EP/N024966/1.

\section{References}

[1] McDonald KC, Saunders KEA, Geddes JR. Sleep problems and suicide associated with mood instability in the Adult Psychiatric Morbidity Survey, 2007. Aust New Zeal J Psychiatry 2017:1-7.

[2] Anderson IM, Haddad PM, Scott J. Bipolar disorder. BMJ 2012;345:8508.

[3] Lieb K, Zanarini MC, Schmahl C, Linehan MM, Bohus M. Borderline personality disorder. Lancet 2004;364:453-61.

[4] Sztajzel J. Heart rate variability: A noninvasive electrocardiographic method to measure the autonomic nervous system. Swiss Med Wkly 2004;134:514-22.

[5] McCraty R, Shaffer F. Heart rate Variability: new perspectives on physiological Mechanisms, assessment of self-regulatory Capacity, and Health risk. Glob Adv Heal Med 2015;4:45-61.

[6] Bassett D. A literature review of heart rate variability in depressive and bipolar disorders. Aust New Zeal J Psychiatry 2015;50:1-9.

[7] Faurholt-jepsen M, Kessing LV, Munkholm K. Neuroscience and Biobehavioral Reviews Heart rate variability in bipolar disorder: A systematic review and. Neurosci Biobehav Rev 2017;73:68-80.

Koenig J, Kemp AH, Feeling NR, Thayer JF, Kaess M. Resting state vagal tone in borderline personality disorder: A meta-analysis. Prog NeuroPsychopharmacology Biol Psychiatry 2016;64:18-26.

[9] Dishman RK, Nakamura Y, Garcia ME, Thompson RW, Dunn AL, Blair SN. Heart rate variability, trait anxiety, and perceived stress among physically fit men and women. Int J Psychophysiol 2000;37:121-33.

[10] Sloan RP, Schwarz E, McKinley PS, Weinstein M, Love G, Ryff C, et al. Vagally-mediated heart rate variability and indices of well-being: Results of a nationally representative study. Heal Psychol 2017;36:73-81.

[11] Kreibig SD. Autonomic nervous system activity in emotion: A review. Biol Psychol 2010;84:394-421.

[12] Yoshino K, Matsuko K. Correlation between mood and heart rate variability indices during daily life. Health (Irvine Calif) 2011;3:553-6.

[13] Palmius N, Tsanas A, Saunders KEA, Bilderbeck AC, Geddes JR, Goodwin GM, et al. Detecting Bipolar Depression from Geographic Location Data. IEEE Trans Biomed Eng 2016;PP:1.

[14] Tsanas A, Saunders KEA, Bilderbeck AC, Palmius N, Goodwin GM, De Vos M. Clinical Insight into latent variables of psychiatric questionnaires for mood symptom self-assessment. JMIR Ment Heal 2017;4:e15.

[15] Carr O, Saunders KEA, Tsanas A, Palmius N, Geddes JR, Foster R, et al. Variability in phase and amplitude of diurnal rhythms is related to variation of mood in bipolar and borderline personality disorder. In Submission 2017.

Address for correspondence: Oliver Carr, Institute of Biomedical Engineering, University of Oxford, OX3 7DQ oliver.carr@eng.ox.ac.uk 